\title{
Amplitude and Frequency Control of a Vibratory Pile Driver
}

\author{
Christopher M. Bingham, Member, IEEE, David A. Stone, Member, IEEE, Nigel Schofield, David Howe, and \\ David Peel
}

\begin{abstract}
This paper describes the digital control of a vibratory pile driver in which the vibration is generated via two tandem pairs of electrically driven, geared, contra-rotating eccentrics. Experimental results are included to show the controller-induced system dynamics for a variety of load conditions, and to highlight the fact that, if the relative phase of the eccentric pairs is not controlled, the natural tendency at high excitation frequency is for the pile driver to operate with a low vibration amplitude. An analytical technique for identifying the system parameters is presented, and analytical performance predictions are compared with experimental results. Analysis of the power flow in the system shows that, although significant power transfer occurs between the two electrical drives, the net power dissipation during pile driving is relatively low.
\end{abstract}

Index Terms-Brushless rotating machines, digital control, load-flow analysis, multiple-input multiple-output systems, system modeling, vibration control.

\section{INTRODUCTION}

W HILE THE USE of piles by the construction industry has been common practice for many years, recent population increases has led to the requirement to build larger structures on less stable sites, with piles being used to reenforce structural foundations. Currently, there are three main categories of pile-driving equipment: impact hammers, vibratory units, and hydraulic presses [1]. Impact hammers rely on hydraulically lifting a ram (up to 40 tons) to a predetermined height (up to $2 \mathrm{~m}$ ) before allowing it to fall under its own weight onto the head of the pile, [2]. While being the most prevalent technology, environmental concerns, regarding the transmission of vibrations through the soil to adjacent structures, and acoustic noise emissions, are leading to the increased use of hydraulic presses and vibratory units. Hydraulic presses rely on jacking the pile against a reaction force, thereby smoothly forcing the pile into the ground. Although this technique generates low levels of vibration and noise, its application is limited to particular types of soil. Vibratory pile drivers, on the other hand, are much more widely applicable, since they exploit the fact that friction between two moving bodies may be significantly reduced if either, or both, are vibrated. Thus, vibration of a pile induces fluidization or liquefaction of the surrounding soil, with the result that it may be driven into the soil under its own weight. Traditionally, vibration is achieved by clamping a pile driver having tandem

Manuscript received October 1, 1998; revised November 23, 1999. Abstract published on the Internet March 12, 2000.

The authors are with the Department of Electronic and Electrical Engineering, University of Sheffield, Sheffield, S1 3JD, U.K. (e-mail c.bingham@sheffield.ac.uk).

Publisher Item Identifier S 0278-0046(00)04750-X. pairs of contra-rotating eccentric masses to the pile, as shown in Fig. 1(a). Rotation of the eccentrics at a constant speed results in sinusoidally time-varying inertial forces and the vertical vibration of the pile, Fig. 1(b). The vibration frequency could be varied by controlling the speed of rotation of the eccentrics, while the amplitude could be controlled by varying the relative phase displacement of the tandem eccentric pairs.

Previous attempts at electromechanical actuation to produce controlled vibration without the need for eccentric masses (which require overrated bearings to accommodate the unbalanced forces) have resulted in the development of novel topologies of electric machines, [3]. Termed "rolling rotor" machines, the external rotor is free to roll on the inside surface of the casing, the vibration being produced by the eccentric movement of the external rotor. Although performance has been verified experimentally, difficulties in scaling up laboratory prototypes to industrial units have prevented the dissemination of the technology. Consequently, therefore, the predominant technology to date consists of two pairs of eccentrics driven by a single, fixed-speed, hydraulic motor, hydraulic power for the system being generated remotely by a diesel-driven hydraulic power pack, and fed to the vibrator through long, high-pressure hoses. The upper vibration frequency of such units is often limited to $25 \mathrm{~Hz}$, and the total eccentric moment is fixed throughout the pile-driving duty cycle as a consequence of a gearbox between the eccentric masses. Significant operational problems which exist with such limits include:

- generation and transmission of large, and potentially damaging, vibrations to adjacent structures, if pile/soil resonances are induced when the fixed eccentric moment is accelerated to the operational frequency, and subsequently decelerated, during both startup and shutdown of the pile driver;

- ineffective pile driving, as a consequence of the fixed vibration amplitude and frequency compacting certain types of soil;

- environmental pollution, due to the inevitable leakage of some hydraulic oil.

In the vibratory pile-driver system described in this paper, the resonance problem is overcome by operating with a phase displacement of $180^{\circ}$ between upper and lower -pairs of eccentrics, to obtain zero net vibration, during both run-up and run-down, while the other problems are addressed by driving each pair of contra-rotating eccentric masses by a variable-speed electric motor. A significant advantage is that these can extend the upper vibration frequency to $50 \mathrm{~Hz}$, offering the potential for significantly increasing the speed of pile driving and reducing 


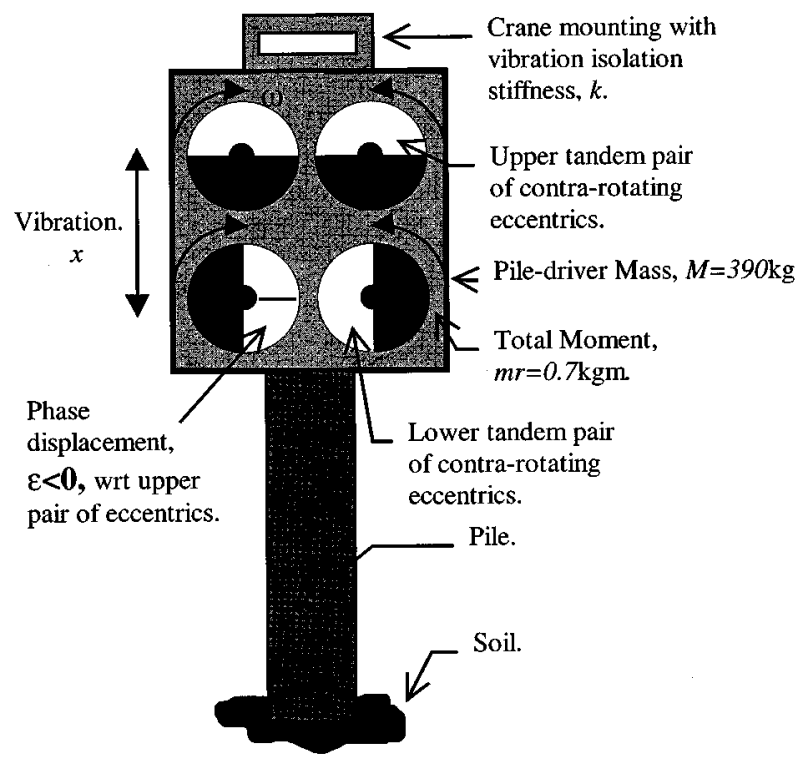

(a)

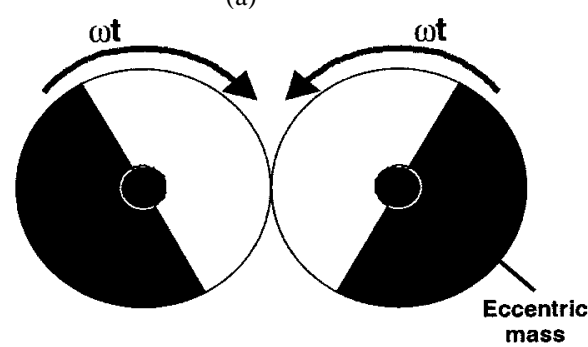

mass

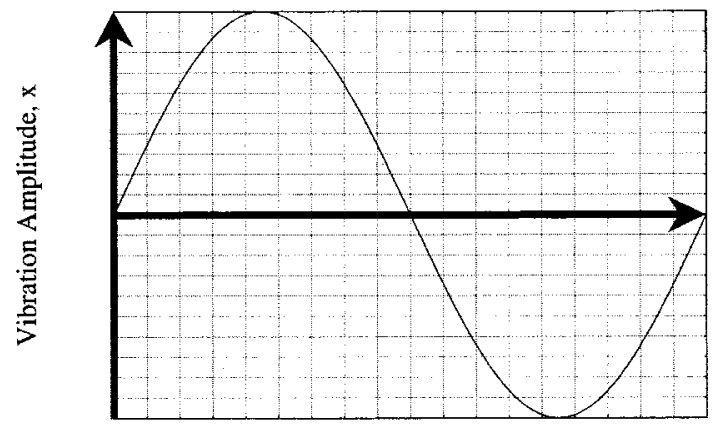

Time

(b)

Fig. 1. (a) Vertical vibration of pile using two pairs of tandem contra-rotating eccentrics. (b) Contra-rotating eccentrics and resultant uniplanar vibration.

the transmission of vibrations into the surrounding environment. Typically, the total eccentric moment in the largest vibrator units is around $100 \mathrm{~kg} \cdot \mathrm{m}$. However, in the prototype unit described in this paper, each eccentric pair had an eccentric moment of $0.7 \mathrm{~kg} \cdot \mathrm{m}$. The eccentrics were accommodated in oil lubrication chambers into which excess oil could be added to experimentally simulate the loading of the vibrator unit which would occur during pile driving.

In order to determine the torque requirement for the two drive motors, industrial two-pole $50-\mathrm{Hz}$ induction motors, each having a continuous rating of $7.5 \mathrm{~kW}$, were initially employed. However, the induction motor drives were torque limited at
$24 \mathrm{~N} \cdot \mathrm{m}$, which limited the maximum operating frequency to around $25 \mathrm{~Hz}$. Nevertheless, by extrapolating results, the peak torque requirement of each drive for $50-\mathrm{Hz}$ operation was estimated as $100 \mathrm{~N} \cdot \mathrm{m}$. Further, although the initial tests had highlighted a significant disparity in the required ratings of the induction motors for driving the upper and lower eccentric pairs, the prototype pile driver was subsequently equipped with two identically rated brushless dc drives. Thus, each eccentric pair was coupled to a custom-designed $30-\mathrm{kW}$ water-cooled brushless dc motor, having a nominal torque rating of $100 \mathrm{~N} \cdot \mathrm{m}$ at $3000 \mathrm{r} / \mathrm{min}$, and supplied from a current-controlled insulated gate bipolar transistor (IGBT) converter via a common mains rectified dc link of $600 \mathrm{~V}$. The common dc link facilitated power circulation in the system, so as to increase the overall efficiency of the pile-driver unit.

The two brushless dc motor/drive units were controlled using a TMS320C50 hardware development platform, which also performed diagnostic and supervisory tasks. Optical shaft encoders provided position feedback. Hence, for each pair of eccentrics, as well as commutation information for the brushless motors, their relative phase displacement, and the phase displacement with respect to the body of the pile driver, could be calculated by the controller in real time (see Fig. 2).

\section{SYSTEM DYNAMICS}

If the inertial moment of each tandem eccentric pair is $m r$, where $m$ is the effective mass of the eccentric-pair rotating at a radius $r$, then, with a relative phase displacement $\varepsilon$ between the tandem pairs, the magnitude of the resultant disturbance force is given by

$$
F_{r}=m r \omega^{2} \cdot \sqrt{2+2 \cdot \cos (\varepsilon)}
$$

where $\omega$ is the constant angular velocity of the eccentric masses. Thus, the excitation force increases with the square of the angular velocity. The dynamic behavior of the complete pile-driver system, embracing the pile driver, pile, and soil, is complex, and has yet to be fully expounded. However, the ensuing analysis examines the system dynamics under the assumption that a second-order mode exists which dominates the system behavior. The model takes the form of a mass-spring viscous damper system in which the mass $M$ includes the mass of the pile driver together with the mass $m$ of each eccentric pair, and the mass of the attached pile; $k$ represents the mounting stiffness, and $c$ is a viscous damping coefficient which represents the pile resistance loading. Under this assumption, the dynamics of the pile driver can be expressed as

$$
\begin{aligned}
(M-2 m) \ddot{x}+m \cdot & \frac{d^{2}}{d t^{2}}\left(x+r \cdot e^{j \omega t}\right) \\
& +m \cdot \frac{d^{2}}{d t^{2}}\left(x+r \cdot e^{j(\omega t-\varepsilon)}\right)=-k x-c \dot{x} .
\end{aligned}
$$

Taking the real part of the complex quantities, corresponding to the vertical displacement $x$, the equation reduces to

$$
M \cdot \ddot{x}+c \cdot \dot{x}+k \cdot x=F_{r} \cdot e^{j(\omega t-\varepsilon / 2)}=F_{x}
$$

where $F_{x}$ is the resulting force produced by the eccentrics. Denoting 


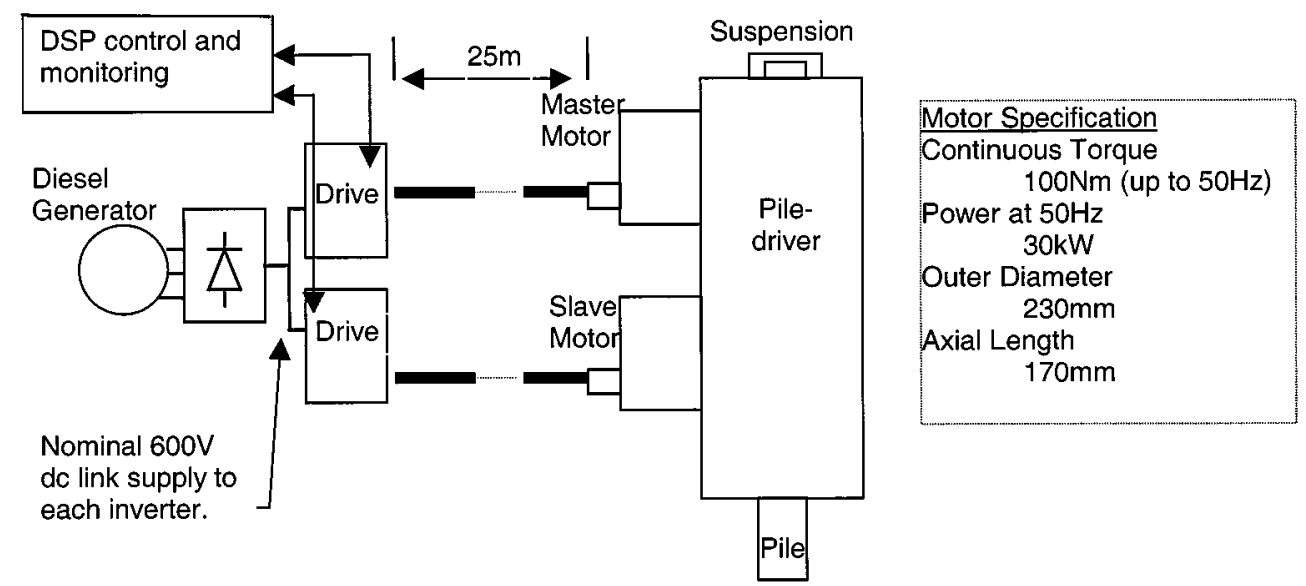

Fig. 2. System schematic and motor parameters.

$$
\begin{aligned}
K & =\frac{m r \cdot \sqrt{2+2 \cdot \cos (\varepsilon)}}{k} \\
\omega_{n} & =\sqrt{\frac{k}{M}} \\
\zeta & =\frac{c}{2 \cdot \omega_{n} \cdot M}
\end{aligned}
$$

the frequency response of the system due to the sinusoidally varying inertial force input is obtained

$$
x(j \omega)=\frac{K \cdot \omega^{2}}{\left(1-\frac{\omega^{2}}{\omega_{n}^{2}}\right)+j \cdot 2 \zeta \cdot \frac{\omega}{\omega_{n}}}
$$

which is analogous in form to the voltage across an inductor in a classical series-resonant second-order $L C R$ circuit. Consequently, the magnitude of the frequency response is given by

$$
|x(j \omega)|=\frac{\left|K \cdot \omega^{2}\right|}{\sqrt{\left(1-\frac{\omega^{2}}{\omega_{n}^{2}}\right)^{2}+4 \zeta^{2}\left(\frac{\omega}{\omega_{n}}\right)^{2}}}
$$

and the frequency at which the maximum amplitude of vibration occurs (as a function of damping coefficient) is

$$
\omega^{2}=\omega_{\mathrm{res}}^{2}=\frac{\omega_{n}^{2}}{1-2 \zeta^{2}}
$$

Substituting the resonant frequency (4) into . (3), the amplitude at resonance $X_{p}$ becomes

$$
X_{p}=\frac{1}{2} \cdot \frac{K \cdot \omega_{\mathrm{res}}^{2}\left(1-2 \cdot \zeta^{2}\right)}{\zeta \cdot \sqrt{1-\zeta^{2}}}, \quad \text { where } 0<\zeta<\frac{1}{\sqrt{2}} .
$$

If the mechanical resonant frequency is much lower than the nominal operating frequency, the amplitude of vibration at high frequency can be estimated from the frequency response equation, (2). For $\omega \gg \omega_{n},(2)$ reduces to

$$
\begin{aligned}
X_{\mathrm{hf}} & =-K \cdot \omega_{n}^{2}=-\frac{m r}{k} \cdot \sqrt{2+2 \cos (\varepsilon)} \cdot \frac{k}{M} \\
& =-\frac{m r}{M} \cdot \sqrt{2+2 \cos (\varepsilon)}
\end{aligned}
$$

where $X_{\text {hf }}$ denotes the amplitude of vibration for a particular phase separation $\varepsilon$ between the contra-rotating eccentric pairs, when operating at high frequencies (relative to $\omega_{n}$ ). If the pile driver is operated with both pairs of eccentrics in phase, i.e., $\varepsilon=$ 0 (maximum vibration), the high-frequency vibration amplitude tends to the constant value $X_{\mathbf{h f m a x}}$, where, from (6)

$$
X_{\mathrm{hfmax}}=K_{\varepsilon=0} \cdot \omega_{n}^{2}=\frac{2 m r}{M} .
$$

Therefore, under the assumption that a second-order mode dominates the dynamic behavior over the frequency range of interest, and that the operating frequency is much higher than the mechanical resonant frequency, (7) shows that the maximum amplitude of vibration is proportional to the ratio of the total eccentric moment to the total mass of the system.

\section{POWER CirCulation}

The power input to the vibratory system is highly dependent on the operating speed, the vibration amplitude, the phase separation between the eccentric pairs, and the pile resistance loading. For a constant operating speed, three components of power flow may be derived:

- a net steady power, dissipated at the damper;

- an oscillatory potential energy component in the eccentrics;

- an oscillatory kinetic energy component in the eccentrics. The corresponding input power components for the upper pair of eccentrics is given by

$$
\begin{aligned}
P_{1 i}=\frac{1}{2} \cdot m \omega^{3} r X_{o} \sin \left(\varphi_{1}\right)+ & m g r \omega \sin (\omega t) \\
& +\frac{1}{2} \cdot m \omega^{3} r X_{o} \sin \left(2 \omega t-\varphi_{1}\right)
\end{aligned}
$$

and, similarly, for the lower eccentric pair

$$
\begin{aligned}
P_{2 i}=\frac{1}{2} \cdot m \omega^{3} r X_{o} \sin \left(\varphi_{2}\right) & +m g r \omega \sin (\omega t-\varepsilon) \\
& +\frac{1}{2} \cdot m \omega^{3} r X_{o} \sin \left(2(\omega t-\varepsilon)-\varphi_{2}\right)
\end{aligned}
$$


where $\varepsilon=\varphi_{1}-\varphi_{2}, X_{o}$ is the amplitude of vibration, and $\varphi_{1}$ and $\varphi_{2}$ are the phase angles between the body of the pile driver and the upper and lower pairs of eccentrics, respectively.

During actual pile driving, the frequency of vibration is generally much higher than the damped mechanical resonant frequency of the system. The first and third terms of the input power equations then become dominant, since the potential term due to gravity increases only in direct proportion to the vibration frequency. Under these conditions, the energy flows largely comprise oscillatory components at the second harmonic frequency of vibration.

A major consideration in the application of independent drive systems to vibratory pile drivers is the division of the total input power between the drives for the upper and lower eccentric pairs. For small phase differences between the eccentric pairs, and at low vibration frequencies, the power input requirements are similar. However, at a sufficiently high frequency, when the upper eccentric pair has a phase lead over the lower pair, while there is still a net power input to the lower eccentric pair, there is a net power output from the upper eccentric pair. When the operational frequency is equal to the natural frequency of the mechanical system and the phase displacement between the eccentric pairs is $90^{\circ}$, the power inputs to the upper and lower eccentrics are equal. Increasing the operating frequency further, while maintaining $90^{\circ}$ phase separation, again results in a net power input to the lower eccentrics and a net power output from the upper eccentric pair. For larger phase separations $(\varepsilon \rightarrow$ $180^{\circ}$ ) and operating frequencies above the natural frequency, there is also a net power input to the lower eccentrics and a net power output from the upper eccentrics. Therefore, power input to the lower eccentrics and power output from the upper eccentrics constitutes the normal operating mode of power flow.

\section{DRIVE SyStem LOAD TORQUE ANALYSIS}

In order to estimate the load-torque variations on the electrical drives during operation, it is assumed that the pile driver generates a sinusoidally time-varying vibration of amplitude $X_{o}$, the instantaneous vertical displacement and acceleration of the pile being given by

$$
\begin{aligned}
& x(t)=X_{0} \cdot \cos \left(\omega t-\phi_{1}\right) \\
& \ddot{x}(t)=-\omega^{2} \cdot X_{o} \cdot \cos \left(\omega t-\phi_{1}\right)
\end{aligned}
$$

respectively, where

$$
\phi_{1}=\frac{\varepsilon}{2}+\tan ^{-1}\left(\frac{\omega \omega}{k-M \omega^{2}}\right) .
$$

For the upper eccentric pair, the load torque on the drive shaft due to the vertical components of force acting on the system mass is, for constant speed operation, given by

$$
\begin{array}{r}
T_{t}=\frac{1}{2} \cdot m r \omega^{2} \cdot X_{o}\left(\sin \left(2 \omega t-\phi_{1}\right)+\sin \left(\phi_{1}\right)\right) \\
+m r g \cdot \sin (\omega t)
\end{array}
$$

which is consistent with the previous power flow analysis.

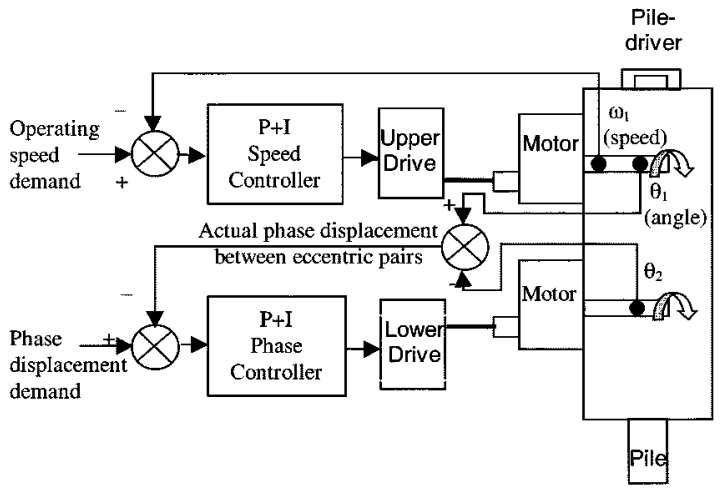

Fig. 3. Initial controller arrangement.

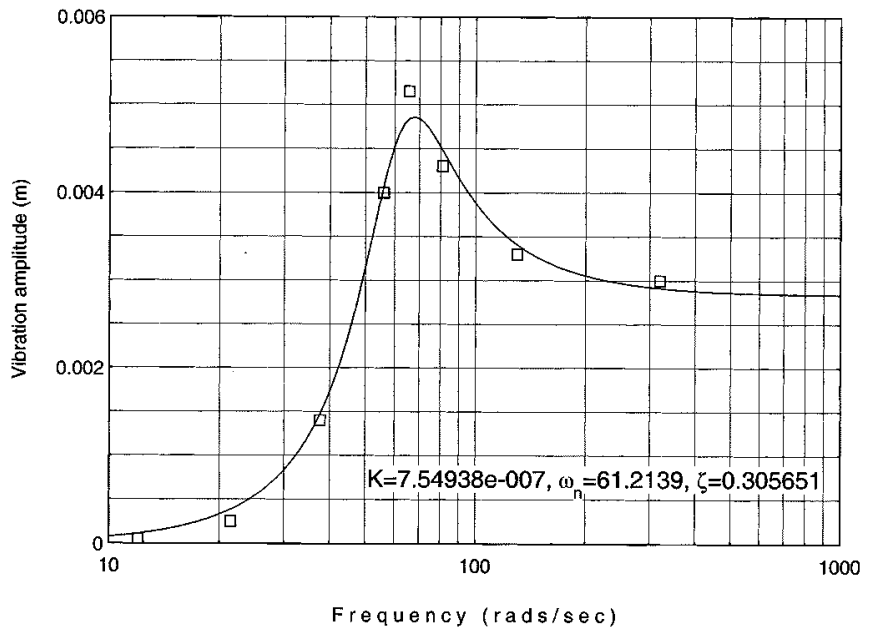

Fig. 4. Variation of maximum vibration amplitude with operating frequency.

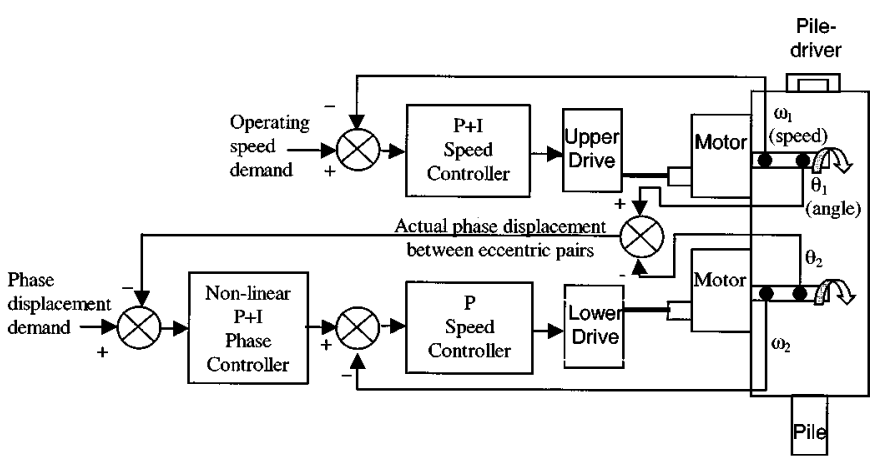

Fig. 5. Nonlinear controller architecture.

The load torque imposed on the drive is, therefore, the sum of components at the first and second harmonic frequencies of rotation (associated with potential and kinetic energy changes, respectively, in the eccentrics), and a frequency dependent steady-state value (associated with driving the damped load). A similar relationship exists for the drive to the lower eccentric pair. At the nominal maximum operating frequency of $314 \mathrm{rad} / \mathrm{s}$ $(50 \mathrm{~Hz})$, therefore, torque disturbances at $628 \mathrm{rad} / \mathrm{s}$ are present. The production of sinusoidally varying vibrations relies on the ability of the controller to reject these load disturbances on the drives and, hence, to promote constant-speed operation. 


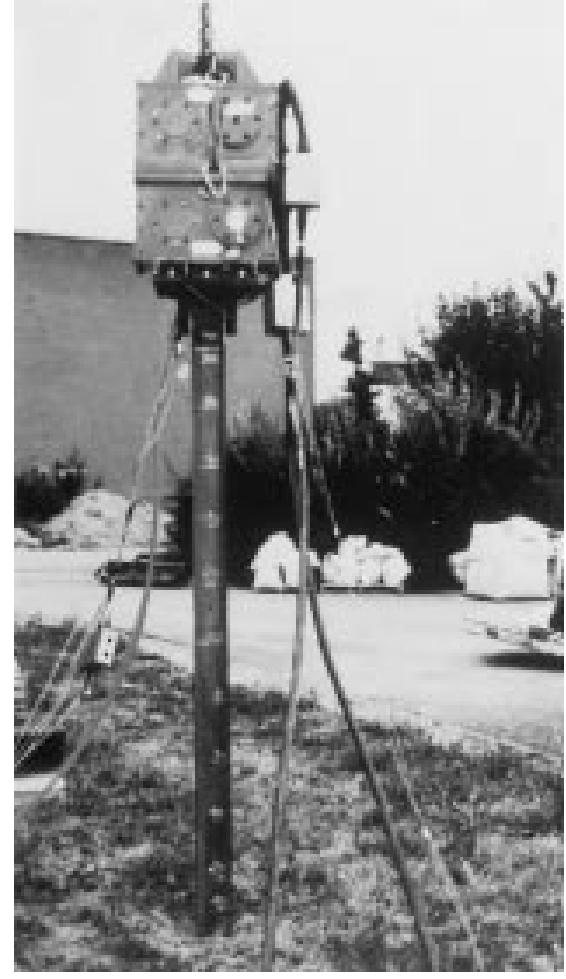

Fig. 6. Prototype vibratory pile driver.

\section{LineAR CONTROLLER DESIGN}

\section{A. Linear Control 1}

An operational requirement of the pile driver is to accelerate the tandem eccentric pairs up to the desired operating frequency whilst maintaining them in antiphase and, thereafter, to control the phase displacement between the eccentric pairs so as to obtain a desired amplitude of vibration and, subsequently, to decelerate with the eccentric pairs again in antiphase. Two relevant (and intuitive) controller inputs are, therefore, speed (operating frequency) and the phase displacement demands. Initial experimental trials were undertaken using a controller on the upper eccentric pair to provide the desired operating speed (and, hence, vibration frequency), and a phase controller on the lower eccentric pair which allowed the lower eccentric pair to "follow" the upper eccentric pair with a desired phase displacement. The control structure is shown in Fig. 3.

The controller, along with diagnostic and supervisory procedures, was implemented on a TMS320C50 digital signal processor (DSP) development platform, with external interfaces to the drive systems provided by custom D/A, A/D, and digital I/O hardware, designed in house. The relative position of the eccentric pairs is measured using incremental optical encoders attached to the drive shafts.

Linear proportional + integral $(\mathrm{P}+\mathrm{I})$ algorithms were implemented for both the speed and phase controllers. This structure provided control of the speed of the eccentrics up to $3000 \mathrm{r} / \mathrm{min}$ $(50 \mathrm{~Hz})$ and the ability to fully control the phase displacement between the eccentric pairs from $0^{\circ}$ to $180^{\circ}$. However, since the speed of the lower eccentric pair was only indirectly controlled, via the phase displacement controller, it meant that the speed of the lower eccentric pair was only loosely coupled to the speed of the upper eccentrics. Exogenous disturbances in the drive systems, and noise in the electronic circuitry, corrupted the control demands. The degradation of the control signals, along with the sequential control structure, meant that performance robustness was significantly impaired, so that both speed and phase synchronization could not be accurately maintained over long operational periods.

\section{B. Linear Control 2}

Due to the dynamic behavior of the pile driver, as described in previous sections, the brushless dc motors which drive the eccentrics are subject to significant load-torque fluctuations. Moreover, the high degree of interaction between the mechanical subsystems causes the modal and disturbance dynamics stemming from the eccentric masses and the motion of the pile driver to be strongly coupled. In particular, oscillatory disturbance torques at the first and second harmonic frequencies of vibration are present, which impose severe demands on the control of the drives.

Mechanically, the pile driver can be operated with a relatively low, and constant, mean torque demand on the motors, which will generally lead to moderate oscillations in the speed of the eccentrics, rather than large torque oscillations associated with constant-speed operation. This, however, makes control of the shaft phase separation, which will have an oscillatory component, more difficult.

Methods for accounting for eccentric disturbance components in multivariable control algorithms include transmission zero assignment [4], observers [5], and high-bandwidth controllers which generally incorporate high gain terms. However, to date, their application to account for the mechanical coupling of multiple eccentric disturbances has not been considered.

During constant speed operation at the maximum operating angular frequency of $314 \mathrm{rads} / \mathrm{s}$ (三3000 r/min), harmonic components of load-torque disturbance up to $628 \mathrm{rads} / \mathrm{s}$ are present. Therefore, the assignment of imaginary axis transmission zeros, at $\pm j 314$ and $\pm j 628 \mathrm{rads} / \mathrm{s}$, would act to reduce the effects of the disturbances on the constant-speed operation of the drives. However, investigations indicated that, for this application, the technique is very problematic, since deviation from the nominal zero-assignment operating frequency (which will occur in practice) would significantly reduce the rejection properties.

An alternative for the rejection of disturbance components in systems is to use observers to estimate the load torque and to apply feedforward corrective control action. However, due to the relatively high frequency of the load-torque fluctuations in this application, a digitally implemented observer would require a sampling frequency in excess of $1 \mathrm{kHz}$. This, coupled with the relatively slow electromechanical modes of the system, would necessitate high numerical accuracy in order to realize the observer. Dynamic scaling on fixed-point processors is a timeconsuming task, which ultimately compromises resolution. The control methodology presented here relies on the implementation of a controller whose bandwidth is sufficient to provide corrective action for the torque disturbances. However, large gain terms are necessary for such control action and, hence, high control activity results. 


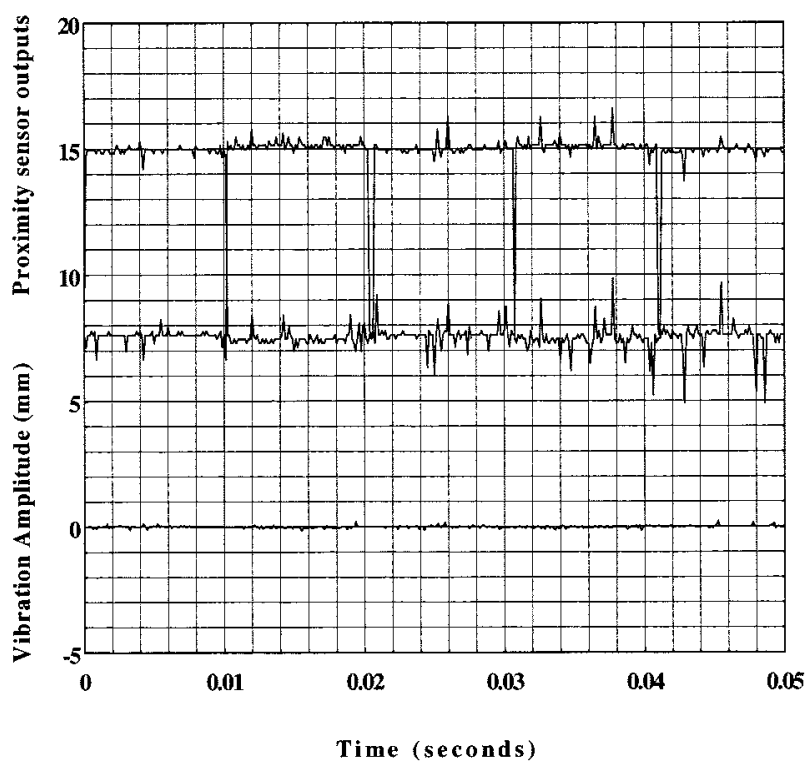

(a)

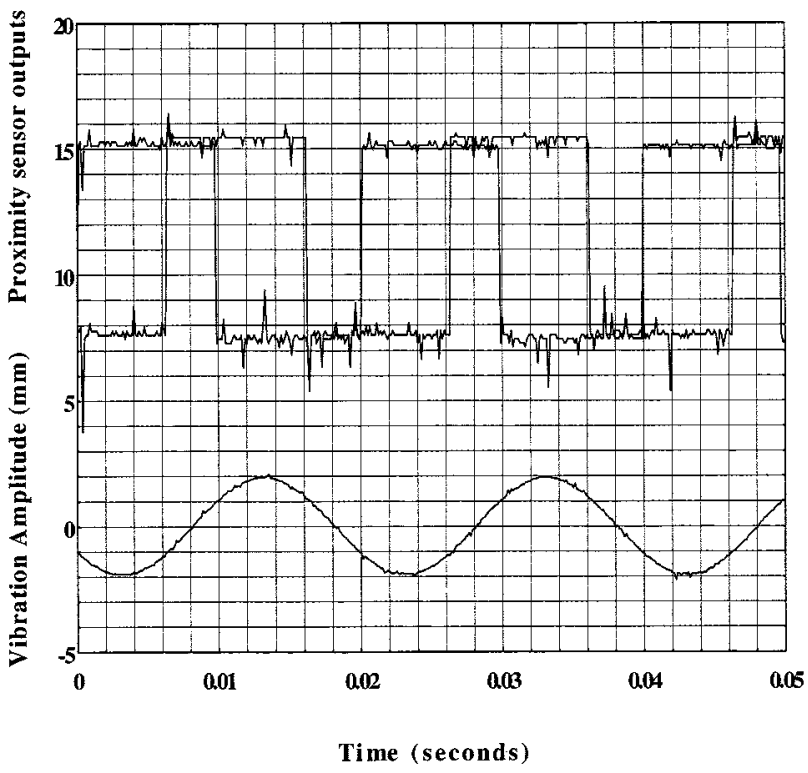

(b)

Fig. 7. $50-\mathrm{Hz}$ operation of vibratory pile driver. (a) Zero vibration amplitude. (b) 4-mm vibration amplitude.

Based on the experience gained from the initial trials described previously, various other control structures have been assessed, all of which are based on a master/slave drive format. The control structure to be described assumes that the upper pair of eccentrics acts as the master, and dictates the frequency of operation of the pile driver (similar to the previous case). The controller for the upper eccentric pair is, therefore, required to ensure minimum deviation between the demanded operating frequency and the actual rotational frequency of the upper eccentric pair. The lower eccentric pair acts as a slave, and aims to maintain a demanded phase lag with respect to the master eccentric pair. As the slave eccentric pair is also required to maintain the same rotational speed as the master pair, an additional speed controller is applied to the slave subsystem.

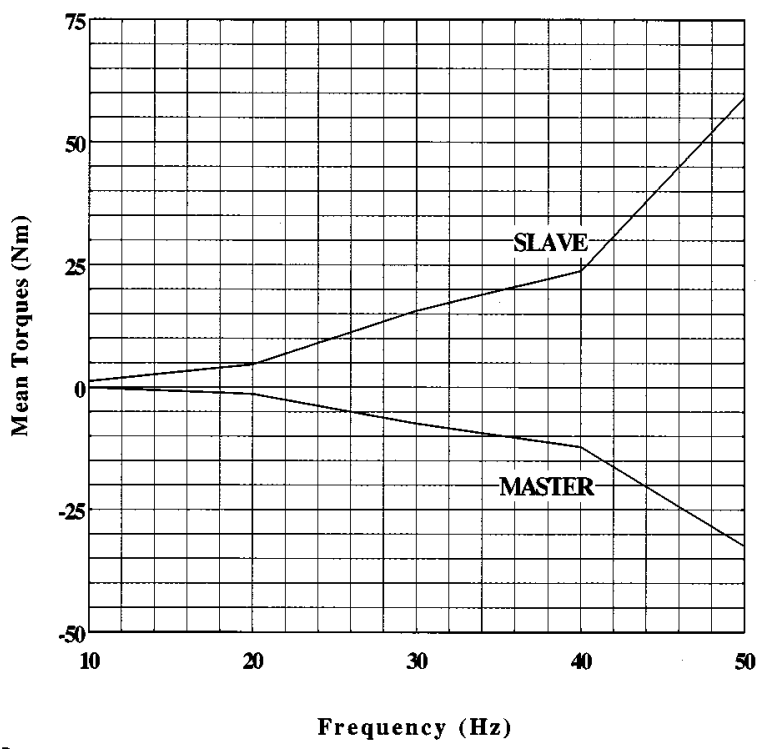

Fig. 8. Torque components associated with master/slave drives for $90^{\circ}$ phase displacement.

Due to the finite amount of available control action, and the need to provide phase synchronization via control of the slave, the speed controller developed for the slave has a relatively low bandwidth. The inertial forces due to the rotation of the eccentrics increase in proportion to the square of the operating frequency. Since the forces which are produced at low speed are relatively small, simple $\mathrm{P}+\mathrm{I}$ control action is adequate for phase synchronization. As the operating speed is increased, however, a significant increase in the system gains is required to maintain synchronization. While experimental trials have demonstrated a great improvement in performance compared to the previous control structure, this strategy, again, does not provide adequate control over the full operating range of the pile driver.

Although lacking in performance robustness, this control methodology facilitated the characterization of the system dynamics. For example, Fig. 4 shows the variation of the amplitude of vibration of the pile driver with operating frequency, when the eccentric pairs are in phase (i.e., maximum vibration condition). A curve fit to the data based on the frequency response magnitude equation (3) is superimposed. The estimated closed-loop resonant frequency, damping ratio, and gain of the system are $61.2 \mathrm{rad} / \mathrm{s}, 0.3$, and $7.55 e-7 \mathrm{~m} / \mathrm{N}$, respectively.

Under the assumption that good speed control of the eccentric pairs is obtained, the overall system dynamics, in terms of the vibration amplitude, are adequate to estimate the mechanical dynamic variables of interest. It is, therefore, assumed that $\omega_{n} \approx$ $\omega_{n \mathrm{cl}}, \zeta \approx \zeta_{\mathrm{cl}}$ and $K \approx K_{\mathrm{cl}}$, where $\omega_{n \mathrm{cl}}, \zeta_{\mathrm{cl}}$, and $K_{\mathrm{cl}}$, are the actual closed-loop resonant frequency, damping ratio, and gain of the system, respectively. The damping ratio is estimated from the ratio of the maximum high-frequency amplitude of vibration $X_{\text {hfmax }}$ and the vibration amplitude at the resonant frequency, $X_{p}$. From $(5) \rightarrow(7)$

$$
\frac{X_{\mathrm{hf}}}{X_{p}}=\frac{K \cdot \omega_{n}^{2}}{\frac{1}{2} \cdot \frac{K \cdot \omega_{n}^{2}}{\zeta \cdot \sqrt{1-\zeta^{2}}}}=2 \cdot \zeta \cdot \sqrt{1-\zeta^{2}}=\frac{2.9}{5.2}=0.56 .
$$




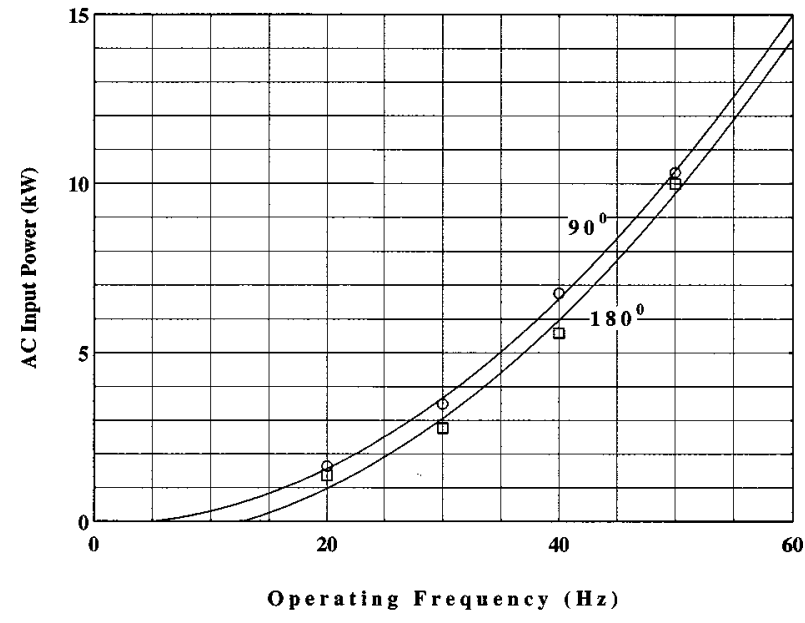

(a)

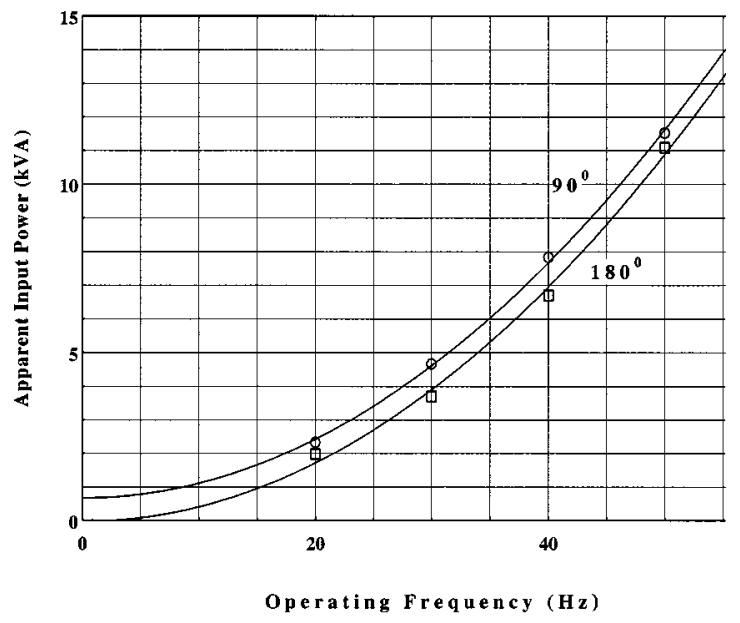

(b)

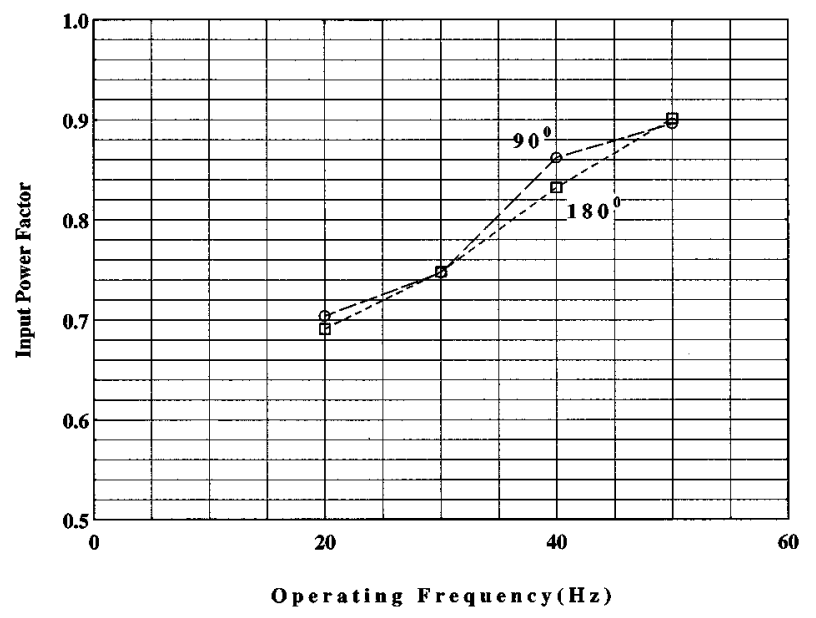

(c)

Fig. 9. System power dissipation for various operating conditions.

Solving the equation results in a number of solutions for the damping factor $\zeta$. However, only one has practical significance: $\zeta=0.29$. From the experimental data, the resonant frequency is estimated to be $66 \mathrm{rads} / \mathrm{s}$, and (4) and (7), therefore, give $\omega_{n}=$ $60 \mathrm{rads} / \mathrm{s}$ and $K=8 e-7$, which are in good agreement with the curve-fit data derived from Fig. 4.

From (1), and noting that the maximum eccentric moment $m r$ of each eccentric pair is $0.7 \mathrm{~kg} \cdot \mathrm{m}$, and the mass $M$ of the system is $400 \mathrm{~kg}, c$ and $k$ are calculated to be $14039 \mathrm{~N} \cdot \mathrm{s} / \mathrm{m}$ and $1444804 \mathrm{~N} / \mathrm{m}$, respectively.

\section{NONLINEAR CONTROL}

Problems encountered with the preceding linear control strategies led to the adoption of a nonlinear $\mathrm{P}+\mathrm{I}$ methodology, whereby the phase control action was modulated as a function of operating speed. The phase control structure was formed empirically by observation

$$
\mathrm{op}(\text { volts })=\left(\varepsilon_{d}-\varepsilon\right) \cdot\left(2+\frac{\omega}{128}\right) \cdot \frac{1}{8}+\frac{1}{2} \int\left(\varepsilon_{d}-\varepsilon\right) \cdot d t
$$

where $\varepsilon_{d}$ is the desired relative phase displacement between the eccentric pairs (see Fig. 5). The nonlinear control structure provides both speed and phase control of the eccentric masses over the full range of operational frequencies and phase displacements and, hence, facilitated experimental trials, the results of which are described below.

\section{EXPERIMENTAL RESULTS}

Experimental results have been obtained from the prototype electromagnetically actuated vibratory pile driver shown in Fig. 6, in which the phase displacement between the eccentric pairs was measured using optical shaft encoders for control purposes and monitored using two inductive proximity sensors, and the vibration was measured using an accelerometer. Fig. 7(a) shows operation at $50 \mathrm{~Hz}$ with zero vibration, the upper and lower eccentric masses being in antiphase. With a controlled phase displacement $\cong 110^{\circ}$ between the eccentric pairs, a peak-to-peak vibration amplitude of $4 \mathrm{~mm}$ results, as shown in Fig. 7(b). 


\section{Relaxation of SpeEd AND Phase Control}

As described previously, the nonlinear controller structure aims to maintain the master eccentric pair rotating at a constant speed, while the slave eccentric pair follows at a desired angular displacement. However, by relaxing the control of the relative phase displacement of the eccentric pairs, the torque rating requirement of the drive systems can be decreased significantly. Although the relaxation of phase control results in oscillations around the desired value of relative phase displacement, the associated speed variation is relatively low.

\section{POWER FLOW}

It can be shown that the vibratory pile driver develops maximum net torque when the phase displacement between the tandem eccentric pairs approaches $90^{\circ}$. The average torques derived from the input drive currents developed by the master (upper eccentric pair) and slave (lower eccentric pair) drive systems at the maximum-torque operating condition are shown in Fig. 8. At $50 \mathrm{~Hz}$, the lower eccentric pair imposes a motoring torque of $60 \mathrm{~N} \cdot \mathrm{m}(18 \mathrm{~kW})$, while the upper eccentric pair imposes a generating torque of $32.5 \mathrm{~N} \cdot \mathrm{m}(10.2 \mathrm{~kW})$, thereby confirming theoretical predictions.

The variation of the net electrical input power, together with the system losses, over the operating frequency range, is shown in Fig. 9(a), for both $90^{\circ}$ (maximum torque) and $180^{\circ}$ (zero vibration) phase separation between the tandem eccentric pairs. Fig. 9(b) shows the corresponding variation of the apparent power and indicates, as in Fig. 9(a), that only a moderate increase occurs over the range of phase separation. Fig. 9(c) shows that the system power factor improves to $0.9 \mathrm{lag}$ at the designed maximum operating frequency, an important criteria in relation to the required on-site generating capacity (note that, under light loading conditions, the majority of the input power is simply to overcome frictional losses).

\section{Mutual Mechanical Coupling}

An inherent feature of a vibratory pile driver which has two independently driven tandem pairs of eccentrics, which stems from the extremely high degree of mechanical coupling between the upper and lower drives, is that synchronization of the tandem eccentric pairs will be maintained, even when one of the drive systems is disabled. By way of example, Fig. 10(a) shows the change of the dc-link currents of the master and slave drives and, therefore, the torques, when the slave is suddenly disabled, while operating at $50 \mathrm{~Hz}$ and with a $120^{\circ}$ phase lag with respect to the master eccentric pair. On disabling the slave drive, the master drive reverts from regenerative operation, to motoring. Although the supply to the slave drive is disabled, the slave eccentric pair continues to rotate as a consequence of the mutual mechanical coupling with the master eccentric pair. Moreover, both sets of eccentric pairs continue to rotate in synchronism, with the phase displacement ultimately increasing toward $180^{\circ}$, as shown by the proximity sensor outputs.

The variation of the phase displacement between the eccentric pairs which results when the slave is disabled and the resultant amplitude of vibration, for various operating frequencies, is

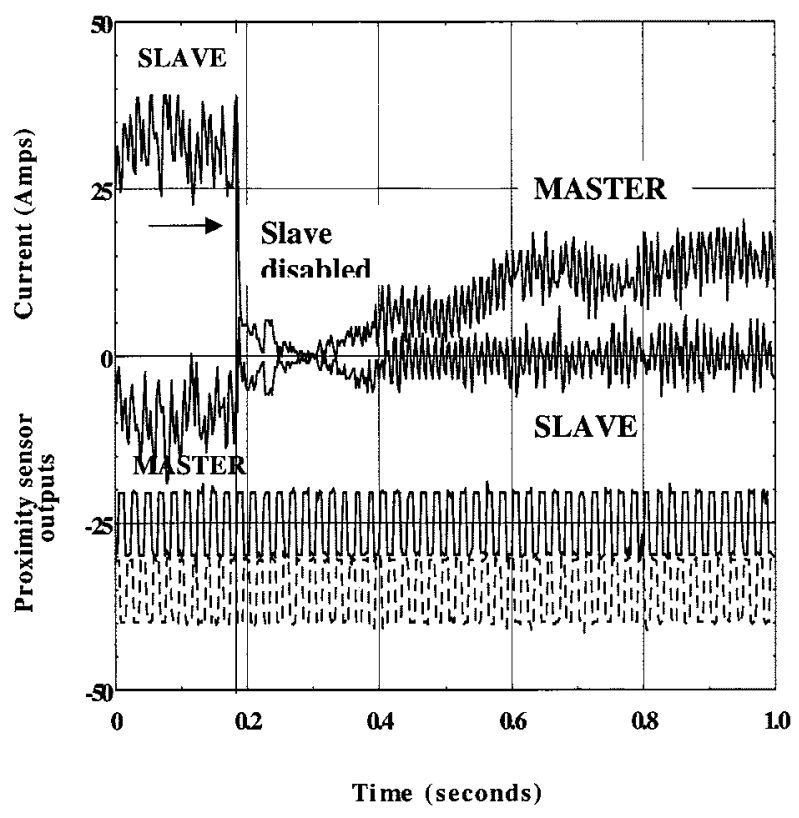

(a)

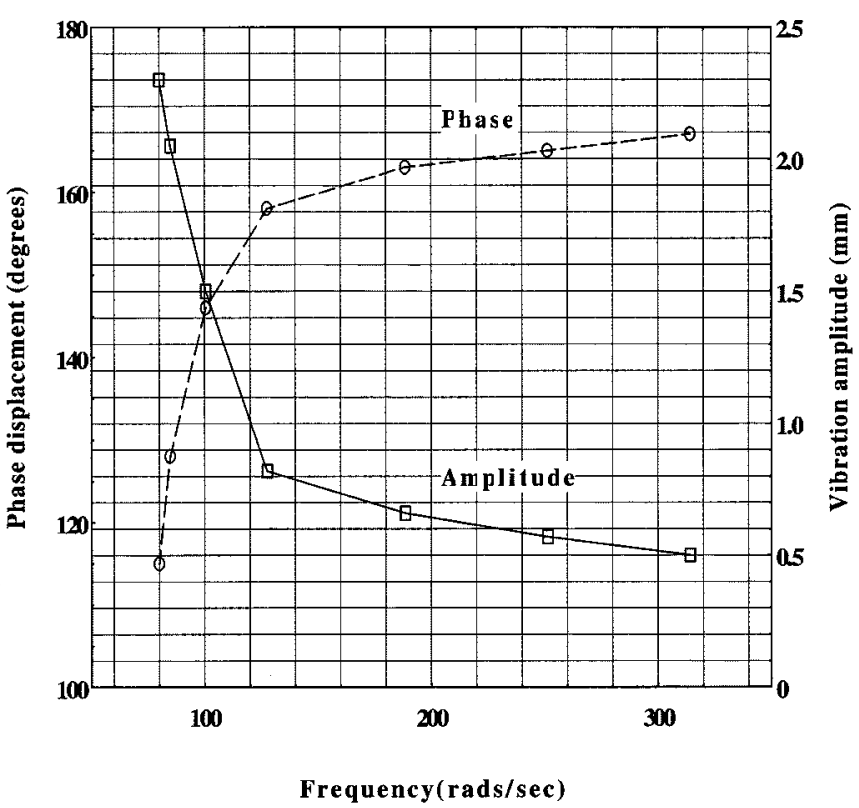

(b)

Fig. 10. (a) Transient current waveforms when slave drive disabled at $t=$ 0.18 s. (b) Steady-state phase displacement of eccentric pairs, and resultant vibration amplitude, with the slave drive disabled.

shown in Fig. 10(b). It is clear that, as the operating frequency approaches the mechanical resonant frequency of the system, the relative phase displacement tends to $90^{\circ}$, and the vibration amplitude increases. However, the natural tendency is for the vibrator to approach a condition of minimum vibration at high frequency, which aids control of the eccentric pairs when zero vibration is required.

\section{CONCLUSION}

This paper has described the development of a prototype electrically actuated vibratory pile driver which can be operated with 
zero vibration up to the maximum operating frequency of $50 \mathrm{~Hz}$ and, thereafter, with the amplitude of vibration controlled. Three control structures have been implemented on a DSP-based hardware platform, and the attributes of each discussed. Power flow measurements have confirmed theoretical predictions, and indicate that, while there is a high power requirement for driving each eccentric pair, the net input power is relatively low, as a consequence of the mutual mechanical interaction. Further, the drive system for the master eccentric pair can be rated at approximately $50 \%$ of that for the slave eccentric pair. Experimental results have also shown that the natural tendency of the pile driver is to revert to a condition of minimum vibration when amplitude control is relinquished at high operating speeds.

Further research has been initiated to remove the mechanical gearing between the pairs of eccentric masses, since this has been found to significantly contribute to acoustic noise emissions. Consequently, each eccentric mass requires independent control by a separate machine, and necessitates synchronized phase and speed control of all four eccentric masses. Clearly, this further complicates the system control problem, since the vibration will not be mechanically constrained to be in the vertical direction, but directed along any desired vector in a two-dimensional vertical plane.

\section{REFERENCES}

[1] W. G. K. Fleming, A. J. Weltnam, M. F. Randolph, and W. K. Elson, Piling Engineering, 2nd ed. New York: Wiley, 1992.

[2] Site Guide to Foundation Construction (Handbook for Young Professionals), Special Pub. 136, Construction Industry Research and Information Association, London, U.K., 1996.

[3] P. R. Mayes, D. A. Stone, D. Howe, Z. Xia, and Z. Zhu, "Tandem rolling rotor actuation systems," in EPE Symp. Electric Drive Design and Applications, Lausanne, Switzerland, 1994, pp. 145-149.

[4] R. V. Patel, "On transmission zeros and dynamic output feedback," IEEE Trans. Automat. Contr., vol. AC-23, pp. 741-742, Aug. 1978.

[5] M. Iwasaki and N. Matsui, "Robust feedforward control of IM with torque feedforward control," IEEE Trans. Ind. Electron., vol. 40, pp. 553-560, Dec. 1993.

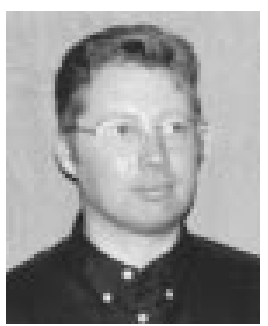

Christopher M. Bingham (M'00) received the B.Eng. degree in electronic systems and control engineering from Sheffield City Polytechnic, Sheffield, U.K., the M.Sc.(Eng.) degree in control systems engineering from the University of Sheffield, Sheffield, U.K., and the Ph.D. degree from Cranfield University, Shrivenham, U.K., in 1989, 1990, and 1994, respectively.

He was a Post-Doctoral Researcher until subsequently taking up a research position at the University of Sheffield. Since 1998, he has been a Lecturer in the Department of Electronic and Electrical Engineering, University of Sheffield. His current research interests include traction control/ antilock braking systems for electric vehicles, electromechanical actuation of flight control surfaces, control of active magnetic bearings for high-speed machines, and sensorless control of brushless machines.

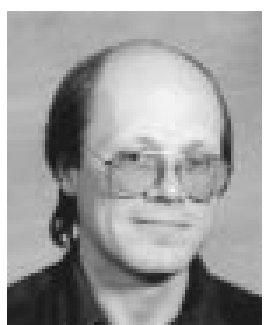

David A. Stone (M'90) received the B.Eng. degree in electronic engineering from the University of Sheffield, Sheffield, U.K., and the Ph.D. degree from Liverpool University, Liverpool, U.K., in 1984 and 1989, respectively.

$\mathrm{He}$ is a member of the academic staff at the University of Sheffield, specializing in power electronics and machine drive systems. His current research interests include hybrid electric vehicles, battery charging, EMC, and novel lamp ballasts for low-pressure fluorescent lamps.

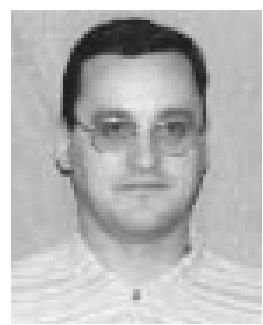

Nigel Schofield received the B.Eng. degree in electrical power engineering and the Ph.D. degree from the University of Sheffield, Sheffield, U.K., in 1990 and 1997, respectively.

During 1993-1995, he was a Senior Experimental Officer in the Department of Electronic and Electrical Engineering, University of Sheffield, before taking up the post of Design Engineer in industry. Since 1997, he has been a Post-Doctoral Researcher in the Electrical Machines and Drives Research Group, Department of Electronic and Electrical Engineering, University of Sheffield, where his research interests include power trains for hybrid electric vehicles and aerospace actuator applications.

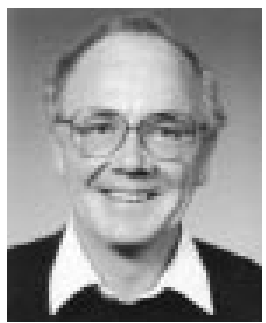

David Howe received the B.Tech. and M.Sc. degrees from the University of Bradford, Bradford, U.K., and the Ph.D. degree from the University of Southampton, Southampton, U.K., in 1966, 1967 and 1974, respectively, all in power engineering.

He has held academic posts at Brunel University and Southampton University and spent a period in industry with NEI Parsons Ltd., working on electromagnetic problems related to turbogenerators. He is currently Lucas Professor of Electrical Engineering, University of Sheffield, Sheffield, U.K., where he heads the Electrical Machines and Drives Research Group. His research activities span all facets of controlled electrical drive systems, with particular emphasis on permanent-magnet excited machines. He is the author of more than 200 publications in the fields of machines, drives, and motion control systems.

Prof. Howe is a Chartered Engineer in the U.K. and a Fellow of the Institution of Electrical Engineers, U.K

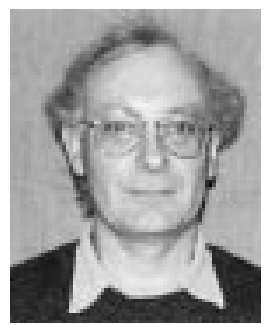

David Peel received the B.Eng. degree in mechanical engineering from the University of Liverpool, Liverpool, U.K., and the D.Phil. degree in applied science from the University of Sussex, Sussex, U.K., in 1968 and 1979 , respectively.

$\mathrm{He}$ has been a longstanding member of, and contributor to, research in the SMMART Laboratory, University of Sheffield, Sheffield, U.K., and has contributed to over 50 publications, particularly in the field of engineering with electrostructured fluids. He currently holds a research post in the Department of Electronic and Electrical Engineering, University of Sheffield, and is a Development Engineer with Magnetic Systems Technology Ltd., Sheffield, U.K., working in the field of hybrid electric vehicles. 